\title{
Breeding Amicable Numbers in Abundance
}

\author{
By W. Borho and H. Hoffmann
}

\begin{abstract}
We give some new methods for the constructive search for amicable number pairs. Our numerical experiments using these methods produced a total of 3501 new amicable pairs of a very special form. They provide some experimental evidence for the infinity of such pairs.
\end{abstract}

\section{Amicable Numbers of Euler's First Form.}

1.1. Historical Remarks on Thabit ibn Kurrah's Formula. Two natural numbers $A$, $B$ are called amicable if each of them is the sum of all proper divisors of the other one. Equivalently, this means that

$$
\sigma(A)=A+B=\sigma(B),
$$

where $\sigma(m)$ denotes the sum of all divisors of $m$. The famous rule of Thabit ibn Kurrah (†Bagdad 801 A.D.) states that

$$
A=2^{n} \cdot r_{1} \cdot r_{2}, \quad \text { and } \quad B=2^{n} \cdot s
$$

are amicable numbers, in the case that

$$
\begin{aligned}
& r_{1}=3 \cdot 2^{n-1}-1, \quad r_{2}=3 \cdot 2^{n}-1, \quad \text { and } \\
& s=\left(r_{1}+1\right)\left(r_{2}+1\right)-1=9 \cdot 2^{2 n-1}-1
\end{aligned}
$$

are prime numbers. For example, $n=2$ gives the amicable pair $A=2^{2} \cdot 5 \cdot 11$, $B=2^{2} \cdot 71$ attributed to the legendary Pythagoras (500 B.C.) by Iamblichos ( 300 A.D.). Two further examples are obtained for $n=4$, resp. $n=7$, as discovered in the early 14th century by Ibn al-Banna $\bar{a}^{\prime}$ in Marakesh, and also by Kamaladdin Fārisī in Bagdad, according to [12], [1], [11], resp. in the 17th century, by Muhammad Bāqir Yazdi in Iran, see [1], [11]. Note that until recently, these first examples of amicable numbers, and also Thabit's rule, had been attributed to Fermat (1636), and Descartes (1638). So much for our little updating of the early history of the subject (cf., [2], [5]).

1.2. A New Analogue of Thabit's Formula. Let us now state an analogue of Thabit's theorem (1.1) which seems to be new:

TheOREM. Let $n$ be a positive integer, and choose $\beta, 0<\beta<n$, such that with $g=2^{n-\beta}+1$, the number

$$
r_{1}=2^{\beta} \cdot g-1
$$

Received September 30, 1984; revised December 19, 1984.

1980 Mathematics Subject Classification. Primary 10A40; Secondary 10A25, 10--04. 
is prime. Now choose $\alpha, 0<\alpha<n$, such that

$$
\begin{gathered}
p=2^{\alpha}+\left(2^{n+1}-1\right) g, \\
r_{2}=2^{n-\alpha} g p-1, \quad \text { and } \\
s=\left(r_{1}+1\right)\left(r_{2}+1\right)-1=2^{n-\alpha+\beta} \cdot g^{2} p-1
\end{gathered}
$$

are also prime. Then

$$
A=2^{n} p r_{1} r_{2} \text { and } B=2^{n} p s
$$

are amicable numbers.

In fact, by the assumptions of the theorem, the reader may verify (1) by straightforward calculations. For example, $n=2$ and $\alpha=\beta=1$ give the amicable pair $A=2^{2} \cdot 23 \cdot 5 \cdot 137$ and $B=2^{2} \cdot 23 \cdot 827$, which was discovered by L. Euler without using such explicit formulae. To avoid confusion, let us mention at this point that another analogue of Thabit's rule was introduced in [3], and further investigated in [13], [5]. Note that the "Thabit rules" of [3], to which we shall come back briefly in Subsection 2.5 of the present paper, are of a quite different nature than the above theorem.

1.3. Euler's Generalization of Thabit's Formula. Thabit's explicit formulae (3) have been generalized by L. Euler to exhaust all amicable pairs of the form (2) with primes $r_{1}, r_{2}, s$ as follows: If for $g=2^{n-\beta}+1$ with some $\beta, 0<\beta<n$, the numbers

$$
\begin{aligned}
& r_{1}=2^{\beta} \cdot g-1, \quad r_{2}=2^{n} \cdot g-1, \quad \text { and } \\
& s=\left(r_{1}+1\right)\left(r_{2}+1\right)-1=2^{n+\beta} \cdot g^{2}-1
\end{aligned}
$$

are prime, then the numbers (2) are amicable, and this gives all amicable pairs of the form (2) (with primes $r_{1}, r_{2}, s$ ). For example, $\beta=n-1$ gives Thabit's rule. Euler's generalization gives further amicable numbers for $n=8, \beta=1$, resp. $n=40$, $\beta=29$, as discovered by Legendre and Chebyshev, resp. by te Riele [13].

1.4. A New Analogue of Euler's Formula. Let us now state an analogue of Euler's exhaustive formulae (1.3), dealing with amicable numbers of the form (8), which seems to be new.

TheOREM. Let $n, \gamma$ be positive integers with $0<\gamma<n$. Put either

$$
\text { (i) } C=2^{n}+2^{\gamma}, \quad \text { or } \quad \text { (ii) } C=2^{n}\left(2^{n+1}-1\right)+2^{\gamma} \text {. }
$$

Take any factorization of $C=f D$ into two positive factors $f, D$. Whenever the four numbers $p, r_{1}, r_{2}, s$ given below in (9)-(11) are primes, then

$$
A=2^{n} \cdot p \cdot r_{1} r_{2} \text { and } B=2^{n} \cdot p \cdot s
$$

are amicable; and all amicable pairs (8) with $p, r_{1}, r_{2}, s$ odd primes, are obtained in this way. Here,

$$
\begin{array}{ll}
\text { (9) } & p=D+2^{n+1}-1, \\
\text { (10) } & r_{1}=p f-1, \quad r_{2}=\left(r_{1}+1\right) \cdot 2^{n-\gamma}-1 \quad \text { in case (i), resp. }
\end{array}
$$$$
r_{1}=2^{n}+f-1, \quad r_{2}=p\left(2^{n}+f\right) \cdot 2^{n-\gamma}-1 \quad \text { in case (ii), and }
$$$$
s=\left(r_{1}+1\right)\left(r_{2}+1\right)-1 \text {. }
$$

For example, $n=8, \gamma=7$, and $f=2^{3} \cdot 31$ give E. J. Lee's amicable pair [9]

$$
A=2^{8} \cdot 1039 \cdot 503 \cdot 1047311, \quad B=2^{8} \cdot 1039 \cdot 527845247 .
$$


If we choose for the factor $f$ a power of two in case (ii), then we obtain Theorem 1.2 as a special case of the present Theorem 1.4. On the other hand, Theorem 1.4 will be seen to be itself a special case of an even more general result (Theorem 1.6 below), and this is the way to prove it.

Remark. As pointed out by the referee, we may allow in case (ii) also $n<\gamma<2 n$, provided that the number $f$ has sufficiently many factors 2 to make $f \cdot 2^{n-\gamma}$ integral. For example, $n=7, \gamma=9$, and $f=2^{4} \cdot 7 \cdot 37$ give P. Poulet's amicable pair

$$
A=2^{7} \cdot 263 \cdot 4271 \cdot 280883, \quad B=2^{7} \cdot 263 \cdot 1199936447 .
$$

1.5. Euler's Search Procedure for Amicable Pairs of the Simplest Form. Let us now consider amicable numbers of the form

$$
A=a \cdot r_{1} r_{2}, \quad B=a \cdot s
$$

with primes $r_{1}, r_{2}, s$ not dividing $a$. Here we allow for the common divisor $a$ now an arbitrary number, in place of the 2-power $2^{n}$ in 1.3. As noted by Euler, for purposes of algebraic discussions, this is the simplest form the prime decomposition of an amicable pair may have, and therefore it is sometimes referred to as "Euler's first form" [7]. Although Euler was able to find an additional 13 of them using several clever methods, such pairs later turned out to be relatively rare. On the other hand, they also turned out to be particularly useful, as inputs for certain methods to construct further amicable numbers of different forms, as will be discussed later (2.5).

So for various good reasons, amicable pairs of Euler's first form (12) have been investigated much more extensively, and more systematically, than those of any other form. For a list of investigators and references, we refer to Table 1 below.

\section{TABLE 1}

Documentation of the 98 amicable pairs of Euler's first type presently known to us.

\begin{tabular}{|c|l|c|c|}
\hline year & authors & $\begin{array}{c}\text { number of } \\
\text { new pairs }\end{array}$ & reference \\
\hline$\approx-500$ & Pythagoras & 1 & {$[11]$} \\
$\approx 1300$ & al-Banna' & {$[12]$} \\
& (Fermat 1636$)$ & & {$[1],[11]$} \\
$\approx 1600$ & Yazdi & 1 & {$[10]$} \\
& (Descartes 1638) & 13 & {$[10]$} \\
1750 & Euler & 1 & {$[10]$} \\
1830 & Legendre/Chebychev & 1 & {$[10]$} \\
1984 & Seelhoff & 1 & {$[10]$} \\
1929 & Mason & 4 & {$[10]$} \\
1946 & Poulet/Gerardin & 8 & {$[10]$} \\
1957 & Escott & 8 & {$[9]$ or [10] } \\
1968 & Garcia & 3 & {$[13]$} \\
1974 & Lee & 1 & {$[7]$} \\
1978 & te Riele & 7 & Costello \\
1979 & Borho, Hoffmann & 18 & Table 2 of \\
& Nebgen, Reckow & & this note \\
1984 & Borho/Hoffmann & 30 & \\
& & &
\end{tabular}


In order to find all amicable pairs of the form (12) for a specified numerical value of $a$, one may proceed as follows: Take any factorization of $a^{2}$ into two factors, $a^{2}=d_{1} d_{2}$; whenever the three numbers

$$
\begin{gathered}
r_{i}=\left(d_{i}+\sigma(a)-a\right) /(2 a-\sigma(a)) \text { for } i=1,2, \text { and } \\
s=\left(r_{1}+1\right)\left(r_{2}+1\right)-1
\end{gathered}
$$

are different prime numbers not dividing $a$, then $A=a r_{1} r_{2}, B=a s$ is an amicable pair; and all such pairs are obtained in this way. For more details of this method, which essentially goes back to Euler, we refer to our paper [5], where we used such an "Euler search" to compute the complete list of (exactly 60, as it turns out) amicable pairs of type (12) with $a \leqslant 10^{7}$. Note that the above statements, applied to the case of a 2-power $a=2^{n}$, readily yield Euler's generalization (1.3) of Thabit's explicit formulae.

1.6. A New Analogue of "Euler's Search". Let us now state another method of search for amicable numbers of Euler's first type, which is a more sophisticated analogue of the "Euler search" (1.5), and seems to be new. The idea is to assume that the common factor $a$ contains a simple prime factor $p$, which is another unknown, to be determined along with $r_{1}, r_{2}$, and $s$, after specification of the cofactor $b=a / p$.

THEOREM. Given a natural number $b$, take first any factorization $b^{2}=d_{1} d_{2}$ of $b^{2}$ into twe factors $d_{1}, d_{2}$, and take secondly any factorization $C=f D$ of the number $C$ below into two factors $f, D$; here $C$ is either

$$
\begin{aligned}
& \text { (i) } \quad C:=b+d_{1} \text {, } \\
& \text { or (ii) } \quad C:=b \sigma(b)+d_{1} \cdot(2 b-\sigma(b)) \text {. }
\end{aligned}
$$

If the four numbers $p, r_{1}, r_{2}, s$, where

$$
\begin{aligned}
& \quad p:=(D+\sigma(b)) /(2 b-\sigma(b)), \\
& \text { and } \quad r_{1}:=p f-1, \\
& r_{2}:=b\left(r_{1}+1\right) / d_{1}-1 \quad \text { in case (i), } \\
& \text { resp. } \quad r_{1}:=(\sigma(b)-b+f) /(2 b-\sigma(b)), \quad \text { in case (ii), } \\
& r_{2}:=b p\left(r_{1}+1\right) / d_{1}-1 \\
& \text { and } s:=\left(r_{1}+1\right)\left(r_{2}+1\right)-1,
\end{aligned}
$$

are all prime, pairwise different, and prime to $b$, then

$$
A=b p r_{1} r_{2}, \quad B=b p s
$$

are amicable, and all such amicable pairs are obtained by the above formulae.

This theorem can be proved by applying 1.5 to the case where $a=b p$ with $p$ prime, and not dividing $b$. We leave the verification of the details to the reader. Putting $b=2^{n}$ we obtain Theorem 1.4 as an immediate corollary of the more general theorem above.

By an extensive computer search based on this theorem, we found that in the range $b \leqslant 10^{6}$, and with the largest prime $s \leqslant 10^{14}$, there are exactly 86 amicable pairs of the form (19) considered in the theorem, 56 of which had been known before. The 30 new pairs are listed in Table 2. 


\section{TABLE 2}

The 30 new amicable pairs of Euler's first form $A=a r_{1} r_{2}, B=$ as, found by means of Theorem 1.6.

\begin{tabular}{|c|c|c|c|}
\hline no. & $a=b \cdot p$ & $r_{1} \cdot r_{2}$ & $s$ \\
\hline 1 & $3^{4} \cdot 5 \cdot 11^{3} \cdot 83$ & $331 \cdot 659$ & 219119 \\
\hline 2 & $3^{3} \cdot 5 \cdot 11 \cdot 103 \cdot 109$ & $41 \cdot 471533$ & 19804427 \\
\hline 3 & $3^{4} \cdot 5^{2} \cdot 13 \cdot 769$ & $389 \cdot 124577$ & 48585419 \\
\hline 4 & $3^{2} \cdot 7 \cdot 11 \cdot 13 \cdot 103 \cdot 109$ & $41 \cdot 471533$ & 19804427 \\
\hline 5 & $3^{2} \cdot 5^{3} \cdot 11 \cdot 16349$ & $29 \cdot 1079033$ & 32371019 \\
\hline 6 & $3^{5} \cdot 5 \cdot 13 \cdot 37 \cdot 2663$ & $89 \cdot 47933$ & 4314059 \\
\hline 7 & $3^{3} \cdot 5^{2} \cdot 19 \cdot 37 \cdot 4079$ & $73 \cdot 73421$ & 5433227 \\
\hline 8 & $3^{6} \cdot 7 \cdot 11 \cdot 17 \cdot 101$ & $857 \cdot 165437$ & 141945803 \\
\hline 9 & $2^{6} \cdot 211 \cdot 4219$ & $173 \cdot 1468211$ & 255468887 \\
\hline 10 & $2^{6} \cdot 587 \cdot 5869$ & $83 \cdot 985991$ & 82823327 \\
\hline 11 & $3^{3} \cdot 5 \cdot 13 \cdot 23 \cdot 1103$ & $2417 \cdot 205157$ & 496072043 \\
\hline 12 & $2^{3} \cdot 17^{2} \cdot 307 \cdot 1877$ & $1733 \cdot 11261$ & 19528307 \\
\hline 13 & $3 \cdot 5^{2} \cdot 7 \cdot 23^{2} \cdot 83$ & $1049 \cdot 1844093$ & 1936298699 \\
\hline 14 & $3^{4} \cdot 7^{2} \cdot 11 \cdot 19 \cdot 461$ & $5531 \cdot 38723$ & 214221167 \\
\hline 15 & $3^{3} \cdot 5^{2} \cdot 19 \cdot 31 \cdot 521$ & $15629 \cdot 296969$ & 4641641099 \\
\hline 16 & $2^{7} \cdot 349 \cdot 27919$ & $491 \cdot 6868073$ & 3379092407 \\
\hline 17 & $3^{2} \cdot 5 \cdot 7 \cdot 113 \cdot 1321$ & $379679 \cdot 634079$ & 240747494399 \\
\hline 18 & $3^{3} \cdot 5 \cdot 11^{2} \cdot 43 \cdot 5689$ & $8513 \cdot 1126421$ & 9590356907 \\
\hline 19 & $3^{2} \cdot 5^{2} \cdot 11 \cdot 43 \cdot 8599$ & $2579 \cdot 22185419$ & 57238383599 \\
\hline 20 & $2^{8} \cdot 1259 \cdot 337411$ & $431 \cdot 18220193$ & 7871123807 \\
\hline 21 & $2^{8} \cdot 599 \cdot 3709$ & $28751 \cdot 53320583$ & 1533073431167 \\
\hline 22 & $2^{7} \cdot 347 \cdot 971$ & $72869 \cdot 283027079$ & 20624183319599 \\
\hline 23 & $3 \cdot 5 \cdot 7 \cdot 11 \cdot 521 \cdot 2083$ & $2459 \cdot 394561859$ & 970622175599 \\
\hline 24 & $3 \cdot 5 \cdot 7 \cdot 13 \cdot 67 \cdot 1499$ & $22511 \cdot 438691343$ & 9875819536127 \\
\hline 25 & $3^{2} \cdot 5 \cdot 7 \cdot 107 \cdot 3851$ & $67409 \cdot 259595909$ & 17499360293099 \\
\hline 26 & $2^{7} \cdot 263 \cdot 75743$ & $4733 \cdot 358567361$ & 1697457891707 \\
\hline 27 & $2^{9} \cdot 1039 \cdot 207797$ & $49871 \cdot 2493563$ & 124359023807 \\
\hline 28 & $3^{3} \cdot 5 \cdot 11^{2} \cdot 43 \cdot 4289$ & $279413 \cdot 27869921$ & 7787246385707 \\
\hline 29 & $2^{4} \cdot 37 \cdot 227 \cdot 79549$ & $743 \cdot 13434871511$ & 9995544404927 \\
\hline 30 & $3^{3} \cdot 5 \cdot 11^{2} \cdot 43 \cdot 31583$ & $459 \cdot 36749347139$ & 90403393964399 \\
\hline
\end{tabular}

\section{Advice for Constructing "Breeders", and How to "Breed" Amicable Numbers} from Them.

2.1. Lee's BDE Method [9]. Given natural numbers $a_{1}, a_{2}$ one may determine all amicable pairs of the form

$$
A=a_{1} q, \quad B=a_{2} s_{1} s_{2},
$$

where $q$, resp. $s_{1}, s_{2}\left(s_{1} \neq s_{2}\right)$, are primes not dividing $a_{1}$, resp. $a_{2}$, by solving a bilinear Diophantine equation (BDE) on $s_{1}, s_{2}$ as follows: Take any factorization of the number

$$
(F+D) F+D G=d_{1} d_{2}
$$

into two different natural factors $d_{1}, d_{2}$, where

$$
\begin{aligned}
& F:=\left(\sigma\left(a_{1}\right)-a_{1}\right) \sigma\left(a_{2}\right), \quad G:=a_{1} \sigma\left(a_{1}\right), \\
& D:=a_{2} \sigma\left(a_{1}\right)+a_{1} \sigma\left(a_{2}\right)-\sigma\left(a_{2}\right) \sigma\left(a_{1}\right) .
\end{aligned}
$$

If then, for $i=1,2$,

$$
s_{i}=\left(d_{i}+F\right) / D
$$


are integer, prime, and prime to $a_{2}$, and if also

$$
q=\sigma\left(a_{2}\right) \sigma\left(a_{1}\right)^{-1}\left(s_{1}+1\right)\left(s_{2}+1\right)-1
$$

is prime and prime to $a_{1}$, then we have an amicable pair (20), and this procedure gives all such pairs. The idea of this method goes essentially back to Euler, who formulated - and extensively used - it in several special cases, as did many authors later on. In the present general form, the method seems to be explicitly formulated for the first time in E. J. Lee's paper [9]. For example, the "Euler search" procedure stated in 1.5 is obtained again as the special case $a_{1}=a_{2}=a$.

2.2. te Riele's Trick: Daughter Pairs From Mother Pairs. Most of the amicable numbers currently known have been found by use of some version of the BDE method. A successful search requires two essential ingredients: 1 . clever choices for the input numbers $a_{1}, a_{2}$, and 2. clever handling of a large amount of primality testing. Before powerful primality tests and adequate computing facilities became available, the second point-for a long time-put a severe restriction on the numerical use of the BDE method, because this method tends to lead too soon to too large primes. Since this restriction has been sufficiently removed, the investigators could focus attention on the first point, on the clever choice of inputs. Recently, te Riele [14] has discovered the remarkable efficiency of the following trick: Take the inputs $a_{1}, a_{2}$ from the lists of already known amicable pairs $\left(A_{1}, A_{2}\right)$ by splitting both numbers $A_{i}$ into $A_{i}=a_{i} v_{i}(i=1,2)$, where $v_{i}$ is either 1 , or a large simple prime factor of $A_{i}$. From a list of 1592 known ("mother") pairs, te Riele computed in [14] in this manner 2324 new ("daughter" and "granddaughter") pairs. A substantial portion of these were produced by the special case of his trick (cf. [15, Lemma 1]), where the mother pair $\left(A_{1}, A_{2}\right)$ is of Euler's first form $\left(a r_{1} r_{2}, a s\right)$, and $v_{1}=1, v_{2}=s$. For example, after sending te Riele our 18 new pairs of Euler's first form found in [5], he almost immediately returned to us a list of 455 new amicable pairs derived from these 18 "mother pairs" as "daughter pairs" in this way. Let us restrict our attention here only to this special case, which is particularly nice and particularly productive for two reasons: First, this particular choice of inputs $\left(a_{1}, a_{2}\right)$ allows us to eliminate the divisions in Eqs. (23) of the BDE-method, so that the values for $s_{1}, s_{2}$ are automatically integer, and second, it guarantees a particularly highly factorizable number in Eq. (21), so that an abundance of values for $s_{1}$, $s_{2}, q$ enter the primality testing, and it becomes likely that at least a few of them successfully pass it.

2.3. Amicable Breeders. To improve te Riele's trick further, we take as inputs $\left(a_{1}, a_{2}\right)$ for the BDE method not only data from amicable ("mother") pairs, because these are still relatively rare, but we allow more general inputs $\left(a_{1}, a_{2}\right)$, called "breeder" pairs, which are not that rare.

Definition. A pair of positive integers $a_{1}, a_{2}$ is called a "breeder", if the equations

$$
a_{1}+a_{2} x=\sigma\left(a_{1}\right)=\sigma\left(a_{2}\right)(x+1)
$$

have a positive integer solution $x$.

The idea is that "breeders" may be used to "breed" amicable "daughter pairs" in the same way as te Riele uses his data from amicable pairs, the only difference being that there might not be any "mother pair" now. Taking a breeder $\left(a_{1}, a_{2}\right)$ as input in the BDE-method 2.1 will produce "daughter pairs" of the form $\left(a_{1} q, a_{2} s_{1} s_{2}\right)$ as 
discussed there (for example). Only if the solution $x$ of (25) happens to be a prime not dividing $a_{2}$, then $\left(a_{1}, a_{2} x\right)$ is an amicable pair, and is a "mother pair", in te Riele's terms, of our daughter pairs. So we may say that the "mother pair", if it exists, is breeded from our "breeder" in 0th generation.

Our point is that "breeders" may not only serve as clever inputs for the BDE method, but that they may also-on the other hand-be produced as outputs of the BDE method. Therefore, from one breeder whole generations of other breeders may be "bred" by the BDE method, and this process may then be used to "breed" an abundance of new amicable pairs.

In fact, to construct breeders $\left(b_{1}, b_{2}\right)$ by the BDE method, one simply proceeds as follows: Starting from any input $\left(a_{1}, a_{2}\right)$ as explained in 2.1 , just check that (23) gives two different primes $s_{1}, s_{2}$ prime to $a_{2}$ (but ignore (24) now). Then $b_{1}=a_{2} s_{1} s_{2}, b_{2}=a_{1}$ is already a breeder. Alternatively, one may check whether (24) gives a prime $q$ not dividing $a_{1}$, and (23) gives at least one prime, $s_{1}$ say, not dividing $a_{2}$; if so, then $b_{1}=a_{1} q, b_{2}=a_{2} s_{1}$ will be a breeder. Many variations are possible.

2.4. More General Breeders. More generally, we may define a "breeder of type $(i, j)$ " to be a pair of positive integers $\left(b_{1}, b_{2}\right)$ such that the equations

$$
b_{1} x_{1} x_{2} \cdots x_{i}+b_{2} y_{1} y_{2} \cdots y_{j}=\sigma\left(b_{1}\right) \prod_{\nu=1}^{i}\left(x_{\nu}+1\right)=\sigma\left(b_{2}\right) \prod_{\mu=1}^{j}\left(y_{\mu}+1\right)
$$

have a solution in positive integers $x_{1}, \ldots, x_{i}, y_{1}, \ldots, y_{j}$. Note that "breeders" in the sense of 2.3 are "breeders of type $(0,1)$ " in the present sense. Although we shall discuss in the sequel only these in more detail, let us point out here that breeders of type $(1,1)$, e.g., are of similar interest.* Note that "breeders of type $(0,0)$ " are just amicable pairs (and that type $(1,0)$ is of course equivalent to type $(0,1)$ ). Equation (26) says that if the positive integers $x_{1}, \ldots, x_{i}, y_{1}, \ldots, y_{j}$ happen to be all prime. pairwise different, and not divisors of $b_{1}$, resp. $b_{2}$, then $\left(b_{1} x_{1} \cdots x_{i}, b_{2} y_{1} \cdots y_{j}\right)$ will be an amicable pair. In the special case where $b_{1}=b_{2}=: a$, such an amicable pair is called regular, of Euler type $(i, j)$. In this terminology, the amicable pairs considered in 1.5 are the regular amicable pairs of Euler type $(2,1)$. They are called "of Euler's first form" because $(2,1)$ are the smallest possible values for $(i, j)$ in this situation, as noted by Euler.

2.5. More Special Breeders, and Thabit Rules [3]. A breeder in the sense of 2.3 is called special, if it is of the form $(a u, a)$ with $u$ prime to $a$. For example, an amicable pair of Euler's first form 1.5 (12) gives rise to a special breeder, with $u=r_{1} r_{2}$ the product of two primes. However, our search procedures (1.5 and 1.6 modified by omitting the primality check for $s$, cf. 2.3) produced roughly seven times more special breeders than amicable pairs of Euler's first type.

Let us point out that already in the paper [3], special breeders in the above sense (resp. in particular amicable pairs of Euler's first form) were used to "breed" new amicable pairs by a method quite different from te Riele's trick (2.2), by constructing so-called "Thabit rules" (cf. loc. cit., Theorems 2 and 4, resp. in particular Theorem 3).

\footnotetext{
*We are presently performing some numerical experiments with type $(1,1)$ breeders, and we intend to report the results in a sequel to the present paper.
} 
THEOREM [3]. Given a special breeder (au,a), assume that

$$
t:=u+\sigma(u)
$$

is a prime not dividing $a$. Then the following ("Thabit") rule holds for $n=1,2,3, \ldots$

$$
A=a u t^{n}\left[t^{n}(u+1)-1\right], \quad B=a t^{n}\left[t^{n}(u+1)(t-u)-1\right]
$$

are amicable, whenever the numbers in square brackets are primes prime to a.

So each special breeder gives rise to a "Thabit rule" whenever (27) gives a prime $t$ (it essentially never happens that $t$ then divides $a$ ). In addition to the 67 Thabit rules previously known (see [3], [13], [7], [5]), we found 34 new ones in this way, as a by-product of our search procedure reported in 1.6 (which was exhaustive in the range $b \leqslant 10^{6}, s \leqslant 10^{14}$, notation 1.6). Since some readers may wish to use them in a search for very large amicable pairs, we list these new rules in Table 3 below.

TABLE 3

The 34 new Thabit rules (notation Theorem 2.5) found by means of Theorem 1.6 ( $c f .2 .5$ ).

\begin{tabular}{|r|l|l|r|}
\hline no. & $a=b \cdot p$ & $u=r_{1} \cdot r_{2}$ & \multicolumn{1}{c|}{$t$} \\
\hline 1 & $3^{3} \cdot 5^{2} \cdot 31^{2} \cdot 19$ & $557 \cdot 10601$ & 11820673 \\
2 & $3^{3} \cdot 7^{2} \cdot 13 \cdot 19 \cdot 43$ & $1091 \cdot 20123$ & 43929601 \\
3 & $3^{5} \cdot 7 \cdot 13 \cdot 17 \cdot 41$ & $3137 \cdot 6561557$ & 41173773313 \\
4 & $2^{8} \cdot 65951$ & $257 \cdot 68061431$ & 35051637223 \\
5 & $3^{4} \cdot 5^{2} \cdot 13 \cdot 769$ & $389 \cdot 124577$ & 97045873 \\
6 & $3^{3} \cdot 5^{4} \cdot 19 \cdot 71$ & $41 \cdot 19949$ & 1655809 \\
7 & $2^{4} \cdot 47 \cdot 181 \cdot 193$ & $28949 \cdot 1276049$ & 73881990001 \\
8 & $3^{4} \cdot 5 \cdot 11 \cdot 79 \cdot 103$ & $3089 \cdot 7109$ & 43929601 \\
9 & $3^{3} \cdot 5 \cdot 13 \cdot 23 \cdot 919$ & $18379 \cdot 716819$ & 26349568001 \\
10 & $3^{2} \cdot 5 \cdot 7 \cdot 113 \cdot 1399$ & $12203 \cdot 151091$ & 3687690241 \\
11 & $2^{2} \cdot 11 \cdot 29 \cdot 109 \cdot 367$ & $653 \cdot 6960521$ & 9097401601 \\
12 & $3^{3} \cdot 5 \cdot 13 \cdot 23 \cdot 1609$ & $1013 \cdot 543841$ & 1102366721 \\
13 & $2^{6} \cdot 179 \cdot 7517$ & $233 \cdot 7035911$ & 3285770671 \\
14 & $3^{6} \cdot 7 \cdot 11 \cdot 17 \cdot 101$ & $857 \cdot 165437$ & 283725313 \\
15 & $3^{4} \cdot 5 \cdot 11 \cdot 47 \cdot 751$ & $5639 \cdot 4235639$ & 47773777921 \\
16 & $3^{3} \cdot 5^{2} \cdot 19 \cdot 31 \cdot 521$ & $15629 \cdot 296969$ & 9282969601 \\
17 & $3 \cdot 5 \cdot 7 \cdot 13 \cdot 67 \cdot 3109$ & $1741 \cdot 6217$ & 21655553 \\
18 & $3^{2} \cdot 5^{2} \cdot 13 \cdot 101 \cdot 1009$ & $19 \cdot 12107$ & 472193 \\
19 & $3^{4} \cdot 7^{2} \cdot 11 \cdot 19 \cdot 461$ & $5531 \cdot 38723$ & 428398081 \\
20 & $3^{5} \cdot 5 \cdot 13 \cdot 41 \cdot 641$ & $71 \cdot 3153719$ & 450981889 \\
21 & $3^{3} \cdot 5^{2} \cdot 17 \cdot 37 \cdot 1091$ & $1997 \cdot 5007689$ & 20005719553 \\
22 & $2^{6} \cdot 1459 \cdot 5281$ & $71 \cdot 277379243$ & 39665231821 \\
23 & $2^{6} \cdot 139 \cdot 104527$ & $751 \cdot 78604303$ & 118142268161 \\
24 & $2^{3} \cdot 53 \cdot 317 \cdot 7607$ & $11 \cdot 91283$ & 2099521 \\
25 & $2^{8} \cdot 547 \cdot 10939$ & $13463 \cdot 294565391$ & 7931762296921 \\
26 & $3^{5} \cdot 5 \cdot 13 \cdot 37 \cdot 2663$ & $89 \cdot 47933$ & 8580097 \\
27 & $3 \cdot 5 \cdot 7 \cdot 11 \cdot 433 \cdot 3463$ & $103889 \cdot 1142789$ & 237447659521 \\
28 & $2^{5} \cdot 97 \cdot 193 \cdot 3089$ & $3577061 \cdot 7154123$ & 51181479476191 \\
29 & $2^{8} \cdot 523 \cdot 23719$ & $192463 \cdot 8728591$ & 3359870540321 \\
30 & $2^{7} \cdot 257 \cdot 102797$ & $24671 \cdot 1233563$ & 60867723781 \\
31 & $2^{5} \cdot 89 \cdot 223 \cdot 13883$ & $17393 \cdot 241480901$ & 8400396120481 \\
32 & $3^{2} \cdot 5 \cdot 7 \cdot 149 \cdot 361927$ & $173 \cdot 9383319401$ & 3256011832321 \\
33 & $2^{8} \cdot 1259 \cdot 337411$ & $431 \cdot 18220193$ & 15724026991 \\
34 & $2^{5} \cdot 97 \cdot 193 \cdot 301079$ & $1559 \cdot 469683239$ & 1464942024001 \\
\hline
\end{tabular}


2.6. Breeding Amicable Pairs From Special Breeders. As a very special case of the general strategy explained in 2.3 , let us now describe more explicitly, and in more detail, how special breeders may be used to "breed" new amicable pairs.

THEOREM. Let $(a u, a)$ be a special breeder. Take any factorization of

$$
C:=\sigma(u)(u+\sigma(u)-1)
$$

into two different factors $D_{1}, D_{2}\left(C=D_{1} D_{2}\right)$. Then if the numbers

$$
s_{i}=D_{i}+\sigma(u)-1 \quad \text { for } i=1,2 \text {, }
$$

and also

$$
q=u+s_{1}+s_{2}
$$

are primes not dividing $a$, then

$$
\left(a u q, a s_{1} s_{2}\right)
$$

is an amicable pair.

In fact, going into the BDE-method in 2.1 with $a_{1}=a, a_{2}=a$, one may derive formulas (29)-(31) above readily from the general formulae (21)-(24) in 2.1 , using the "breeder condition" (25). In the special case where $s:=\sigma(u)-1$ happens to be a prime not dividing $a$, we will be in a situation with a "mother pair" (au,as), and then the above recipe is equivalent to the one stated by te Riele in [15, Lemma 1], by which he produced about half of his new amicable pairs (cf., 2.2). It turned out in our numerical experiments, that arbitrary "special breeders", used as inputs into this recipe, usually "breed" new amicable pairs at a similarly high rate of fertility.

2.7. Numerical Experiments on Breeding. Let us conclude with a brief summary of our numerical experiments about special breeders $(a u, a)$ with $u=r_{1} r_{2}$ the product of two different primes $r_{1}, r_{2}$. Note that such breeders will breed regular amicable pairs of Euler type $(3,2)$ (notation 2.4). By our previous remarks (cf. 2.5), it is clear how such special breeders may be found by (a trivial modification of) our Euler search procedure (1.5), resp. by the new procedure described in 1.6 , along with the search for amicable pairs of Euler's first type (1.6) and for Thabit rules (2.5). In the range $a \leqslant 10^{7}, r_{1} \leqslant r_{2} \leqslant 10^{12}$ there turned out to be exactly 141 special breeders $\left(a r_{1} r_{2}, a\right)$, in addition to those associated with a "mother pair" (and hence covered by te Riele's investigations). From these special breeders, we derived 1669 amicable pairs by the above recipe (Theorem 2.6), 1604 of which were new. Our new search procedure in 1.6 generated more than 300 further new special breeders of the same type $\left(a r_{1} r_{2}, a\right)$, of which only those 153 with $r_{1} r_{2} \leqslant 10^{12}$ were actually employed for breeding, according to the above recipe. This resulted in a list of another 1867 new amicable pairs. The smallest member of the list, for example, is the pair:

$$
\begin{aligned}
& 2 \cdot 5 \cdot 19^{2} \cdot 37 \cdot 127 \cdot 29 \cdot 4217 \cdot 1889453, \\
& 2 \cdot 5 \cdot 19^{2} \cdot 37 \cdot 127 \cdot 147629 \cdot 1619531
\end{aligned}
$$

From a single special breeder, we have thus bred about a dozen new amicable pairs, in the average. Our "champion breeder" was $(a \cdot 1019 \cdot 391882979$, $a)$, where $a=3^{2} \cdot 5 \cdot 13 \cdot 19 \cdot 73 \cdot 277$, which bred 110 new amicable pairs. For more details about our numerical results, and in particular for the full list of our $30+1604+$ $1867=3501$ new amicable pairs found here, we refer to a forthcoming joint report of H. J. J. te Riele and the authors [6]. 
What, in conclusion, do the results of our numerical experiments about breeding amicable numbers suggest? We obtain about three and a half thousand amicable pairs of a very special type (Euler's type $(3,2)$ ) by a very special recipe. In combination with some analysis of their distribution, we think that this gives some rather convincing numerical evidence to expect that there is an infinity of amicable pairs, even of this particular special type.

2.8. Why "Special" Breeders? As a final point, let us briefly come back to the breeder condition (25) in 2.3. Under a certain mild regularity assumption, we shall now show that such breeders are necessarily "special" in the sense of 2.5 .

Proposition. Assume that $\left(a_{1}, a_{2}\right)$ is a breeder in the sense of 2.3 , and that it "breeds" at least one regular amicable pair $A=a_{1} q, B=a_{2} s_{1} s_{2}$ as in (20) by the process described in 2.1. Then $\left(a_{1}, a_{2}\right)$ is necessarily a special breeder in the sense of 2.5.

Proof. From the regularity of the pair $A, B$ it follows that we must have $a_{1}=a u$, $a_{2}=a v$, with $a, u, v$ pairwise relatively prime to each other. From the breeder condition (25), we have-using the notations as in 2.1:

$$
F:=\left(\sigma\left(a_{1}\right)-a_{1}\right) \sigma\left(a_{2}\right)=a_{2} \sigma\left(a_{2}\right) x=a_{2}\left[\sigma\left(a_{1}\right)-\sigma\left(a_{2}\right)\right],
$$

which gives

$$
D:=a_{2} \sigma\left(a_{1}\right)-F=a_{2} \sigma\left(a_{2}\right),
$$

or also

$$
F=D x \text {. }
$$

Putting this into Eqs. (23), we obtain for $i=1,2$ that

$$
s_{i}=d_{i} / D+x
$$

must be integer, and hence $d_{1} d_{2}$ is divisible by $D^{2}$. Now putting $F=D x$ into Eq. (21) gives

$$
d_{1} d_{2}=x(x+1) D^{2}+D G
$$

hence we must have

$$
d_{1} d_{2} \equiv 0 \equiv D G \bmod D^{2}
$$

Recalling Eq. (22), this is equivalent to

$$
a_{1} \sigma\left(a_{1}\right) \equiv 0 \bmod a_{2} \sigma\left(a_{2}\right) .
$$

Recalling now $a_{1}=a u, a_{2}=a v$ from above, this implies

$$
u \sigma\left(a_{1}\right) \equiv 0 \bmod v .
$$

But since $u, v$ are relatively prime, $v$ must divide $\sigma\left(a_{1}\right)$, which divides $\sigma\left(a_{1} q\right)=$ $\sigma(A)=\sigma(B)=A+B=A+a v s_{1} s_{2}$. It follows that $v$ must also divide $A=a_{1} q=$ auq. By our assumptions, this implies $v=1$, and so proves the proposition. Q.E.D.

Remark. The proposition says that "nonspecial" breeders are usually useless for our purpose. Let us mention here another, more obvious, reason which causes a breeder to be useless for our purpose: More generally, let $\left(a_{1}, a_{2}\right)$ even be an arbitrary type $(i, j)$ breeder in the sense of Subsection 2.4 , and let $c_{k}$ be the greatest common divisor of $a_{k}$ and $\sigma\left(a_{k}\right)$. We observe that then $c_{1}$ must divide $a_{2}$, and $c_{2}$ 
must divide $a_{1}$, or else the breeder would become useless. In fact, any amicable pair $A_{1}$, $A_{2}$ "breeded" from $a_{1}, a_{2}$ should have the form $A_{k}=a_{k} u_{k}(k=1,2)$, with $a_{1} a_{2}$ relatively prime to $u_{1}, u_{2}$, since the "unknown" prime factors of $u_{1} u_{2}$ should be different from the "known" prime factors of $a_{1} a_{2}$. Then Eqs. (1) imply immediately that $c_{1}$ divides $A_{2}=a_{2} u_{2}$, and hence $a_{2}$. Similarly, $c_{2}$ divides $a_{1}$. Needless to say, in numerical experiments one should make sure not to use " useless" breeders.

2.9. A Criterion for Type $(1,1)$ Breeders. By definition 2.4, a pair of natural numbers $a_{1}, a_{2}$ is a type $(1,1)$ breeder, if the equations

$$
a_{1} x+a_{2} y=\sigma\left(a_{1}\right)(x+1)=\sigma\left(a_{2}\right)(y+1)
$$

have a solution in positive integers $x, y$. Let us rewrite the two equations (33) as

$$
a_{1} x-\tau\left(a_{2}\right) y=\sigma\left(a_{2}\right), \quad-\tau\left(a_{1}\right) x+a_{2} y=\sigma\left(a_{1}\right),
$$

where $\tau$ denotes the function $\tau(n):=\sigma(n)-n$. This is an inhomogeneous system of linear equations with determinant

$$
D:=a_{1} a_{2}-\tau\left(a_{1}\right) \tau\left(a_{2}\right)=a_{1} \sigma\left(a_{2}\right)+a_{2} \sigma\left(a_{1}\right)-\sigma\left(a_{1}\right) \sigma\left(a_{2}\right) .
$$

It is therefore solvable if and only if $D \neq 0$, the solutions then being given by

$$
D y=F+G, \quad D x=F^{\prime}+G^{\prime},
$$

where $F, G$ are as in 2.1, and $F^{\prime}, G^{\prime}$ are defined analogously by interchanging $a_{1}$ and $a_{2}$; or in detail:

$$
\begin{aligned}
& F:=\tau\left(a_{1}\right) \sigma\left(a_{2}\right), G:=a_{1} \sigma\left(a_{1}\right), \\
& F^{\prime}:=\tau\left(a_{2}\right) \sigma\left(a_{1}\right), \quad G^{\prime}:=a_{2} \sigma\left(a_{2}\right) .
\end{aligned}
$$

Obviously, the solutions $x, y$ are positive if and only if $D$ is positive. Let us now put

$$
H:=F+D=a_{2} \sigma\left(a_{1}\right), \quad H^{\prime}:=F^{\prime}+D=a_{1} \sigma\left(a_{2}\right),
$$

and rewrite (36) in the equivalent form

$$
\begin{aligned}
& D(y+1)=H+G=\left(a_{1}+a_{2}\right) \sigma\left(a_{1}\right), \\
& D(x+1)=H^{\prime}+G^{\prime}=\left(a_{1}+a_{2}\right) \sigma\left(a_{2}\right) .
\end{aligned}
$$

Then it becomes obvious that the solutions $x, y$ are integers if and only if

$$
\left(a_{1}+a_{2}\right) \sigma\left(a_{i}\right) \equiv 0 \bmod D
$$

for $i=1$ and 2 . This may be written as a single congruence for the greatest common divisor:

$$
\left(a_{1}+a_{2}\right)\left(\sigma\left(a_{1}\right), \sigma\left(a_{2}\right)\right) \equiv 0 \bmod D .
$$

In conclusion, we have proved the following

LEMma. A necessary and sufficient condition for two natural numbers $a_{1}, a_{2}$ to be a type $(1,1)$ breeder is that the determinant $D:=a_{1} a_{2}-\tau\left(a_{1}\right) \tau\left(a_{2}\right)$ be positive, and divides $\left(a_{1}+a_{2}\right)\left(\sigma\left(a_{1}\right), \sigma\left(a_{2}\right)\right)$.

2.10. Breeders Which Are of Types $(0,1)$ and $(1,1)$ Simultaneously. Let us now take for $a_{1}, a_{2}$ a breeder in the sense of 2.3 , that is to say, a type $(1,0)$ breeder in the terminology of 2.4. In this case, it follows from the breeder condition (25) that the determinant $D$ is

$$
D=a_{1} \sigma\left(a_{1}\right)>0 .
$$


Let us now assume that $a_{1}, a_{2}$ are simultaneously also a $(1,1)$ breeder. Then condition (40) with $i=1$, in combination with Eq. (42), implies that necessarily $a_{1}$ must divide $a_{2}$. So let us write $a_{1}=a, a_{2}=a \cdot u$. If we restrict attention to regular amicable pairs, we may assume, in addition, that $a$ and $u$ are relatively prime. So in conclusion, we find that $\left(a_{1}, a_{2}\right)$ must be even a "special breeder" in the sense of 2.5 .

On the other hand, if $(a, a \cdot u)$ is such a special breeder, then $D=a \sigma(a)$, and $\sigma(a)$ divides $\sigma\left(a_{2}\right)=\sigma(a u)=\sigma(a) \sigma(u)$, so that conditions (40) are satisfied for both $i=1,2$. Now Lemma 2.9 implies that $(a, a \cdot u)$ is also a $(1,1)$ breeder. In conclusion, we have shown in particular the following

Proposition. Any special breeder (as defined in 2.5) is a breeder of types $(0,1)$ and $(1,1)$ simultaneously.

Remark. Note that whenever the positive integer solutions $x, y$ of (33) are prime numbers, not dividing $a_{1}, a_{2}$, then the given type $(1,1)$ breeder belongs to an amicable pair (a "mother pair", as te Riele would say), of the form $\left(a_{1} x, a_{2} y\right)$.

Let us point out here, however, that surh is never the case for the special breeders $\left(a_{1}, a_{2}\right)=(a, a \cdot u)$ as considered in the above proposition: In fact, from Eqs. (39) it follows in this case that

$$
D(y+1)=a(u+1) \sigma(a),
$$

and since $D=a \sigma(a)$ by (42), we have $y=u$, and so $y$ is never prime to $a_{2}$ in this case. Thus, such $(1,1)$ breeders never belong to a "mother pair". But let us point out that they nevertheless can be very "fertile" breeders, when used as inputs for the BDE-method (2.1). Numerical experiments shall be reported elsewhere.

Note Added in Proof (August 31,1985). Numerical experiments with type $(1,1)$ breeders, carried out by Stefan Battiato and the first author, generated (in first and second generation) about a thousand amicable pairs so far, 600 of which were new. The most frequent type of these new pairs are $(3,3),(4,2),(4,3)$ or $(3,2)$ (about $80 \%)$, but there are also some of types $(4,4),(5,2),(5,3)$, and about $10 \%$ are of "exotic" type. We are extending these breeding experiments to higher generations. All of these new amicable pairs will be published in [6] as well.

Bergische Universität

Gesamthochschule Wuppertal

FB 7 Mathematik

Gaussstrasse 20

5600 Wuppertal 1, West Germany

1. Alireza Djafari Naini, Geschichte der Zahlentheorie im Orient (im Mittelalter und zu Beginn der Neuzeit unter besonderer Berücksichtigung persischer Mathematiker), Braunschweig, 1982.

2. W. Borно, "Befreundete Zahlen-Ein zweitausend Jahre altes Thema der elementaren Zahlentheorie," in Lebendige Zahlen, Math. Miniaturen, Vol. 1, Birkhäuser Verlag, Basel, 1981.

3. W. Borнo, “On Thabit Ibn Kurrah's formula for amicable numbers,” Math. Comp., v. 26, 1972, pp. $571-578$.

4. W. Borнo, "Some new large primes and amicable numbers," Math. Comp., v. 36, 1981, pp. $303-304$.

5. W. BoRHo, "Große Primzahlen und befreundete Zahlen: Über den Lucas-Test und Thabit-Regeln," Mitt. Math. Ges. Hamburg, v. 11, 1983, pp. 232-256. 
6. W. Borho, H. Hoffmann \& H. J. J. TE Riele, A Table of 7500 Amicable Pairs, CWI-report. (In preparation.)

7. P. J. Costello, “Amicable pairs of Euler's first form,” J. Recreational Math., v. 10, 1978, pp. $183-189$.

8. KamaladDin Fārisī, Tadkirat al-ahbāb fi bayān at-tahābb ( Aufzeichnung der Freunde über die Erklärung der Freundschaft), Bagdad, ca. 1337 (see [1], [11]).

9. E. J. LEE, “Amicable numbers and the bilinear diophantine equation," Math. Comp., v. 22, 1968, pp. 181-187.

10. E. J. LeE \& J. S. Madachy, “The history and discovery of amicable numbers I-III," $J$. Recreational Math., v. 5, 1972, pp. 77-93, 153-173, 231-249.

11. R. RASHED, "Nombres amiables, parties aliquotes et nombres figurés aux XIIIème et XIVème siècles," Arch. Hist. Exact Sci., v. 28, 1983, pp. 107-147.

12. M. Soussi, Un Texte Manuscrit d'Ibn-Al Bannă'AlMarrakusi (1256-1321) sur les Nombres Parfaits, A hondants, Deficients et Amiables, Hamdard National Foundation, Pakistan, Karachi, 1975.

13. H. J. J. TE Riele, “Four large amicable pairs,” Math. Comp., v. 28, 1974, pp. 309-312.

14. H. J. J. TE RIELE, “On generating new amicable pairs from given amicable pairs,” Math. Comp., v. 42, 1984, pp. 219-223.

15. H. J. J. TE RIELE, “New very large amicable pairs,” Journées Arithmétique (Proc. ed. by H. Jager), Springer Lecture Notes in Math., 1984. (To appear.)

16. BĀQIR Muhammad Yazdì, Uyūn al-hisab (Source of Arithmetic), 17th century, Central University Library, Teheran, MS. 464 (see [1], [11]). 